\title{
Executive control over unconscious cognition: attentional sensitization of unconscious information processing
}

\author{
Markus Kiefer* \\ Department of Psychiatry, University of UIm, UIm, Germany
}

\section{Edited by:}

Nicola De Pisapia, University of

Trento, Italy

Reviewed by:

Bruno Breitmeyer, University of Houston, USA

Heiko Reuss,

Julius-Maximilians-University of

Wuerzburg, Germany

*Correspondence:

Markus Kiefer, Department of Psychiatry, Section for Cognitive Electrophysiology, University of UIm, Leimgrubenweg 12, UIm 89075, Germany.

e-mail: markus.kiefer@uni-ulm.de
Unconscious priming is a prototypical example of an automatic process, which is initiated without deliberate intention. Classical theories of automaticity assume that such unconscious automatic processes occur in a purely bottom-up driven fashion independent of executive control mechanisms. In contrast to these classical theories, our attentional sensitization model of unconscious information processing proposes that unconscious processing is susceptible to executive control and is only elicited if the cognitive system is configured accordingly. It is assumed that unconscious processing depends on attentional amplification of task-congruent processing pathways as a function of task sets. This article provides an overview of the latest research on executive control influences on unconscious information processing. I introduce refined theories of automaticity with a particular focus on the attentional sensitization model of unconscious cognition which is specifically developed to account for various attentional influences on different types of unconscious information processing. In support of the attentional sensitization model, empirical evidence is reviewed demonstrating executive control influences on unconscious cognition in the domains of visuo-motor and semantic processing: subliminal priming depends on attentional resources, is susceptible to stimulus expectations and is influenced by action intentions and task sets. This suggests that even unconscious processing is flexible and context-dependent as a function of higher-level executive control settings. I discuss that the assumption of attentional sensitization of unconscious information processing can accommodate conflicting findings regarding the automaticity of processes in many areas of cognition and emotion. This theoretical view has the potential to stimulate future research on executive control of unconscious processing in healthy and clinical populations.

Keywords: unconscious information processing, automatic processes, attentional control, visuo-motor priming, semantic priming, emotional priming, subliminal perception

\section{INTRODUCTION}

Unconscious processes are prototypical examples of automatic processes, which are initiated without deliberate intention (Posner and Snyder, 1975). An important method to measure unconscious processes are subliminal priming (e.g., facilitatory) effects on subsequent decisions or actions on visible targets, which are elicited by masked visual stimuli that are not consciously perceived (Greenwald et al., 1996; Vorberg et al., 2003; Kiefer, 2007). Typically, a pattern or metacontrast mask is presented after-or in the case of pattern masking also before the prime-to prevent its conscious perception (Breitmeyer and Öğmen, 2006). Although these stimuli cannot be consciously perceived, there are meanwhile numerous demonstrations that they trigger cognitive processes at several levels of complexity and thus can influence decisions and actions (for reviews see, Dehaene et al., 2006; Kiefer et al., 2011; Schmidt et al., 2011; Ansorge et al., 2011b; Kunde et al., 2012).

According to classical theories of cognitive control and automaticity (Posner and Snyder, 1975; Schneider and Shiffrin, 1977), such unconscious processes are automatic in the sense that they occur in a purely bottom-up driven fashion independent of attentional control mechanisms. Attentional executive control mechanisms that organize action and thought (Norman and Shallice, 1986; Posner and DiGirolamo, 1998) according to higher-level goals are assumed to be exclusive to the domain of conscious cognition. Classical theories of cognitive control, therefore, propose that only conscious processes depend on capacitylimited attentional resources and can be modulated by executive control. This alleged association of executive control and attention with the domain of conscious cognition has been recently challenged in two respects: (1) Unconscious stimuli influence executive control settings. Several experiments showed that subliminal stimuli can modulate shifts of spatial (Ansorge et al., 2002; Scharlau and Ansorge, 2003) and modality-specific attention (Mattler, 2003, 2005) as well as task-specific control operations (Mattler, 2003, 2005, 2006) and task sets (Reuss et al., 2011; Wokke et al., 2011). (2) Furthermore, the relation between executive control and unconscious processing is bidirectional because top-down factors such as attentional resources, stimulus expectations, action intensions, or task sets, all factors that are typically considered to involve executive control mechanisms (Norman and Shallice, 1986), modulate unconscious stimulus 
processing (Jaśkowski et al., 2003; Ansorge and Neumann, 2005; Kiefer and Martens, 2010; Wokke et al., 2011). Hence, these two lines of research suggest that executive control mechanisms interact with unconscious information processing in several ways and are thus not exclusive to the domain of conscious cognition (Kiefer et al., 2011).

This article provides an overview of the latest research on executive control influences on unconscious information processing. I introduce refined theories of automaticity with a particular focus on the attentional sensitization model of unconscious cognition (Kiefer and Martens, 2010) which is specifically developed to account for various executive control influences on different types of unconscious information processing. In support of these refined theories of automaticity, empirical evidence is presented demonstrating attentional influences on unconscious cognition in the domains of visuo-motor and semantic processing. This suggests that even unconscious processing is flexible and context-dependent as a function of executive control settings.

\section{CLASSICAL VERSUS REFINED THEORIES OF AUTOMATICITY}

The classical view of executive control and automaticity is still influential and pervades current theorizing about automaticity and cognitive control. The core assumption of this view that executive control is exclusive to the domain of conscious cognition while unconscious automatic processes are autonomous (Posner and Snyder, 1975; Schneider and Shiffrin, 1977) implies that a behavioral or neurophysiological effect has to be contextindependent in order to index a "truly automatic" process (Pessoa et al., 2003). Such operational definitions of automaticity, which are essentially influenced by the classical view, can be found in many areas of psychology and neuroscience such as object or face recognition (e.g., Pessoa et al., 2002; e.g., Wiese et al., 2008), action preparation (e.g., Bub and Masson, 2010), and emotional processing (e.g., Pessoa et al., 2002). However, it is difficult to identify processes that actually meet the classical criteria for automaticity because task demands frequently modulate behavioral and neurophysiological effects (see also, Moors and De Houwer, 2006). As almost all kind of cognitive activity has to be classified as "controlled" according to classical criteria, the distinction between strategic and automatic processing becomes practically superfluous. This renders the classical view of automaticity unsatisfactory. Furthermore, if unconscious automatic processing were contextindependent, this would result in a tremendous inflexibility of the cognitive system (Kiefer and Martens, 2010): conscious goal-directed information processing would be massively influenced by various unconscious processes. Demands on conscious executive control would be increased, because the intended action could only be ensured by inhibiting numerous interfering response tendencies induced by unconscious information processing (Botvinick et al., 2001).

Refined theories of automaticity and unconscious processing allow for more flexibility and adaptability of unconscious automatic processing (Neumann, 1990; Naccache et al., 2002; Moors and De Houwer, 2006; Kiefer, 2007; Kiefer and Martens, 2010). These theories posit that unconscious or automatic processing in general depends on a configuration of the cognitive system by attention and task sets. Neumann (1990) proposes in his theory of direct parameter specification (DPS) that unconscious information will only be processed and will influence the motor response to a target stimulus to the extent that it matches current intentions. Similarly, the global workspace model of consciousness by Dehaene and Naccache (2001) explicitly assumes that unconscious processes are susceptible to attentional amplification. Unlike classical theories, refined theories propose that executive control factors such as attention, intentions, and task sets orchestrate the unconscious processing streams toward greater optimization of task performance. Given this dependency on the precise configuration of the cognitive system, the term "conditional automaticity" has been coined (Bargh, 1989; Logan, 1989).

\section{THE ATTENTIONAL SENSITIZATION MODEL OF UNCONSCIOUS COGNITION}

Although previous refined theories of automaticity agree that automatic processes are susceptible to top-down control, they do not account for a number of executive control factors and different forms of automatic processes. The attentional sensitization model of unconscious cognition (Kiefer and Martens, 2010) was developed within this line of research, but aims at explaining the various influences of executive control factors on different forms of unconscious automatic processing. It is proposed that attentional influences originating from task sets enhance taskrelevant unconscious processes while attenuating task-irrelevant unconscious processes. Task sets are defined as an adaptive configuration of the cognitive system which is necessary to efficiently perform a given task (Rogers and Monsell, 1995; Kiesel et al., 2010). The concept of "task set" refers to the immediate computational consequences of pursuing a current goal during task performance that are implemented by executive control mechanisms (Kiesel et al., 2010).

Much as conscious perception is influenced by attentional mechanisms, unconscious cognition is thought to be controlled by top-down signals from prefrontal cortex (Haynes et al., 2007) that increase or decrease the sensitivity of processing pathways for incoming sensory input (Hopfinger et al., 2000, 2001; Bode and Haynes, 2008). Processing in task-relevant pathways is enhanced by increasing the gain of the neurons in the corresponding areas, whereas processing in task-irrelevant pathways is attenuated by a decrease of the gain (Reynolds et al., 2000). Gain is a parameter in neural network modeling, which influences the probability that a neuron fires at a given activation level (Hamker, 2005). Single cell recordings in non-human primates have shown that the likelihood of a neuron firing, given a constant sensory input, is enhanced when the stimulus dimension that is preferentially processed by the neuron is attended to (e.g., Treue and Martínez Trujillo, 1999). Hence, the attentional sensitizing mechanism is thought to gradually enhance and attenuate stimulus processing irrespective of whether the stimulus is consciously perceived or not (Kiefer and Martens, 2010).

Two basic predictions can be derived from the attentional sensitization model: in a manner similar to conscious strategic processes, unconscious automatic processes (1) should depend on available attentional resources, and (2) should be susceptible 
to executive control by currently active task representations. Attentional sensitization of unconscious processing by task representations is achieved by enhancing the sensitivity of task-relevant pathways and by attenuating the sensitivity of task-irrelevant pathways.

Although executive control of both unconscious and conscious processing shares basic computational principles, control of conscious strategic processing is more flexible in several respects. Preemptive control, in which top-down influences are initiated in advance of stimulus presentation, can be exerted for both conscious and unconscious stimulus presentation, whereas only consciously perceived stimuli are susceptible to reactive control in response to ongoing or completed stimulus processing (Ansorge and Horstmann, 2007; Kiefer, 2007; Ansorge et al., 2009, 2011a; Kiefer and Martens, 2010). For that reason, subliminal information cannot be used for determining further strategic processing steps in a deliberate or intentional fashion (Merikle et al., 1995). Executive control of unconscious information processing cannot be exerted intentionally in anticipation or response to subliminal stimuli themselves because individuals are not aware of them by definition. Executive control in the unconscious domain must occur indirectly on the grounds of other representations, whether conscious or unconscious: executive control can be based on a currently active conscious action goal that is internally generated by the individual or externally induced by task instructions (Ansorge and Neumann, 2005). Executive control can also be based on the consciously perceived outcome of overt behavior, which leads to an adjustment of control settings (Jaśkowski et al., 2003). These conscious goal or outcome representations establish or modify task sets that in turn regulate the sensitivity of processing pathways for both conscious and unconscious information. As mentioned at the beginning of this article, control settings are not only intentionally created in a conscious mode, but can also be unconsciously triggered by subliminal stimuli such as attentional or task cues (e.g., Ansorge et al., 2002; Mattler, 2005; Reuss et al., 2011). However, as executive control in the unconscious domain is preemptive, subliminal stimuli that trigger or modify cognitive control settings must be presented before the unconscious process of interest is elicited (e.g., by a subliminal prime). According to the attentional sensitization model, intentional application of control and on-line modification is restricted to conscious strategic processes (see also Dehaene et al., 2006). Finally, executive control of unconscious processing is presumably based on facilitatory influences, that is, it depends on differential attentional sensitization, whereas active inhibition of task-irrelevant information appears to be confined to controlled processing of consciously perceived stimuli (Posner and Snyder, 1975; Neely, 1977; Merikle et al., 1995). Thus, according to the attentional sensitization model conscious stimulus processing, which is traditionally considered to be "strategic," allows for a greater adaptability and flexibility of executive control than unconscious automatic information processing (for a discussion see, Kiefer and Martens, 2010). One can, therefore, distinguish even within the light of refined theories of automaticity strategic and automatic processes although the defining criteria differ from classical theories in several aspects.

\section{EXECUTIVE CONTROL INFLUENCES ON UNCONSCIOUS INFORMATION PROCESSING}

In the upcoming parts of this article, I will review latest evidence demonstrating executive control influences on unconscious visuo-motor and semantic information processing in support of the attentional sensitization model of unconscious cognition.

\section{UNCONSCIOUS VISUO-MOTOR PROCESSING}

Unconscious visuo-motor processing is typically investigated with the masked visuo-motor response priming paradigm (subliminal visuo-motor priming): responses to visual targets are faster, when the masked prime (also a visual stimulus) indicates the same (congruent) rather than a different (incongruent) response (Neumann and Klotz, 1994; Dehaene et al., 1998; Vorberg et al., 2003; Ansorge and Neumann, 2005). In this paradigm, a visual shape is frequently arbitrarily assigned with a motor response (Schmidt et al., 2011). This form of priming depends on visuo-motor processes that give rise to response conflict in the incongruent condition. In line with this interpretation, visuo-motor response priming modulates ERPs over the occipito-parietal scalp in a time window between 200 and $400 \mathrm{~ms}$ (Jaśkowski et al., 2003; Martens et al., 2011). These ERPs most likely arise from the parietal visuo-motor system as identified in a previous functional magnetic resonance imaging (fMRI) study (Wolbers et al., 2006).

In line with the proposed notion of executive control over unconscious processing, subliminal visuo-motor priming effects were shown to be influenced by action intentions and stimulus expectations. The influence of action intentions has been intensively studies by Ansorge and colleagues (e.g., Ansorge et al., 2002, 2010; Ansorge and Neumann, 2005). They found that unconsciously perceived masked primes trigger responses only if they are congruent with the current intentions of a person. Visuomotor priming effects were abolished when task instructions were changed in such a way that primes ceased to be task-relevant. For instance, primes and targets with a similar shape elicited subliminal response priming effects only when the response decision was based on the target's shape (Ansorge and Neumann, 2005). However, when the instruction of the target task was changed such that the response decision was based on the target's color, response priming effects disappeared although primes and targets still exhibited similar or dissimilar shapes (Ansorge and Neumann, 2005). In a comparable experiment, shape or color congruency of masked primes and visible targets only primed target responses, when the corresponding prime feature (e.g., shape feature during shape decisions on the target) was relevant in the target task (Tapia et al., 2010). The task-irrelevant prime feature did not influence responses to targets (see also, Wokke et al., 2011). Similarly, spatial congruency of prime and target words indicating either an elevated (e.g., "above") or a lowered location (e.g., "below") produced priming effects only during a spatial target task, but not during a target task with numbers of high and low numerical magnitude (Ansorge et al., 2010). These findings suggest that action intentions sensitize congruent and desensitize incongruent unconscious processing pathways: it is proposed that an attentional top-down signal enhances unconscious processing of the stimulus dimension that matches the current intention 
(Kiefer and Martens, 2010). Attentional sensitization results in subliminal priming effects on responses to visible targets only for stimulus dimensions that are congruent with the current action intention.

In addition to action intentions, unconscious visuo-motor processing has been shown to depend on stimulus expectations. Stimulus expectations establish an attentional set, which indicates what kind of stimuli are likely to occur within a given situation. Expected subliminal stimuli receive attentional amplification and are more efficiently processed whereas processing of unexpected stimuli is attenuated. It should be noted that expectations cannot be established by unconsciously presented stimuli themselves, but must be formed by consciously perceived stimuli presented within a given context, for instance by the visible target stimuli of a priming paradigm. It has been shown that the type of visible target stimuli included in an experiment strongly influences subliminal priming effects: masked stimuli prime responses only if they are expected and represent possible release conditions for prepared actions to the visible targets (Kunde et al., 2003; Eckstein and Perrig, 2007; Kiesel et al., 2009). Subliminal visuo-motor priming effects elicited by novel primes, which are not presented as targets, are only obtained when they belong to or are at least similar to the attentional set established by the visible targets: for instance, subliminally presented novel numbers prime numerical categorizations of visible numbers only when they are located within the magnitude space spanned by the visible targets (e.g., the prime numbers " 2 " and " 3 " are within the magnitude space spanned by the visible targets " 1 " and " 4 "), but not when they are outside the magnitude space spanned by the visible targets (e.g., the prime numbers " 1 " and " 2 " are outside the magnitude space spanned by the visible targets " 3 " and “4”). Expectancy effects on unconscious visuo-motor priming were also obtained for novel verbal stimuli within a semantic categorization task when the target set size was manipulated (Kiesel et al., 2006). When target set size was large (40 targets) so that a variety of words from different semantic categories was expected, novel word primes elicited visuo-motor priming effects. However, when target set size was small (four targets) so that attention could be focused on a narrow set of stimuli, novel word primes did not produce subliminal priming. These findings demonstrate that stimulus expectations establish an attentional set that sensitizes the corresponding visuo-motor processing pathways for unconscious stimuli. As a result, only expected subliminal stimuli elicit priming effects.

Unconscious visuo-motor processing does not only depend on specific stimulus expectations, but is also influenced by focusing attention in time: subliminal visuo-motor priming in a numerical judgment task was only obtained when the onset of the primetarget pairs was temporally predictable and therefore, attended to (Naccache et al., 2002). Hence, not only the content of the attentional set (specific stimulus expectations), but also the temporal dynamics of attention plays an important role in executive control of unconscious visuo-motor processes.

The mechanisms underlying attentional influences on unconscious visuo-motor processing were further specified in a study using the induction task paradigm (Martens et al., 2011). The induction task paradigm was recently developed (Kiefer and
Martens, 2010) to specifically test the predictions of the attentional sensitization model (see Figure 1A). It allows directly investigating the influence of activated task sets on unconscious information processing, irrespective of action intentions to visible targets or global stimulus expectations. In this paradigm, two different tasks are performed in quick succession: the subliminal priming task is preceded by different classification tasks serving to activate specific task sets. As task sets are active for about $600 \mathrm{~ms}$ after task completion (Rogers and Monsell, 1995), they should influence processing of subsequently presented subliminal primes.

In our study (Martens et al., 2011), the subliminal visuomotor priming task required participants to perform right or left-hand responses to discriminate between geometrical target shapes (e.g., circle or square). The visible target was preceded by a masked prime that either indicated the same or a different motor response, but was never combined with the identical shape to avoid repetition effects. Prior to this subliminal visuo-motor priming task, participants were engaged in a perceptual classification (round vs. elongated object) or in a semantic classification task (living vs. non-living object) designed to induce a specific task set (e.g., a perceptual or semantic task set). These induction tasks were followed immediately by the priming task. According to the proposed attentional sensitization model, visuo-motor priming should benefit from a previous sensitization of visual pathways by the perceptual induction task compared with the semantic induction task. In line with these predictions, behavioral and electrophysiological effects showed a differential modulation of subliminal visuo-motor priming by the induction tasks: visuomotor priming, depending on access to visual shape information, was only observed after the perceptual but not after the semantic induction task (see Figure 1B).

In a continuation of this line of research, the induction task paradigm was used for a fine-grained analysis of task set influences on unconscious visuo-motor response priming. There is evidence that shape and color of visible objects can be attended to and processed independently of each other (Boucart et al., 1995). Based on these findings, the induction task was varied within the perceptual domain to further assess whether the proposed attentional sensitization mechanism not only distinguishes between broad cognitive domains such as visual vs. semantic stimulus attributes but also specifically sensitizes stimulus attributes within the perceptual domain (Zovko and Kiefer, submitted). The effects of a shape decision induction task similar to previous experiments (Kiefer and Martens, 2010) was contrasted with a novel color decision task, in which the hue of colored object picture had to be classified (red vs. blue). In the visuo-motor priming task, participants performed again right or left-hand responses to discriminate between geometrical target shapes (Martens et al., 2011). Occipito-parietal ERP priming effects were only found subsequent to the shape induction task. No such effects were found subsequently to the color induction task. These results show that attentional influences can also occur within perceptual subdomains, such as shape and color attributes. Attentional sensitization thus modulates unconscious visuo-motor processes fine-grained at the level of specific visual object features. 
A

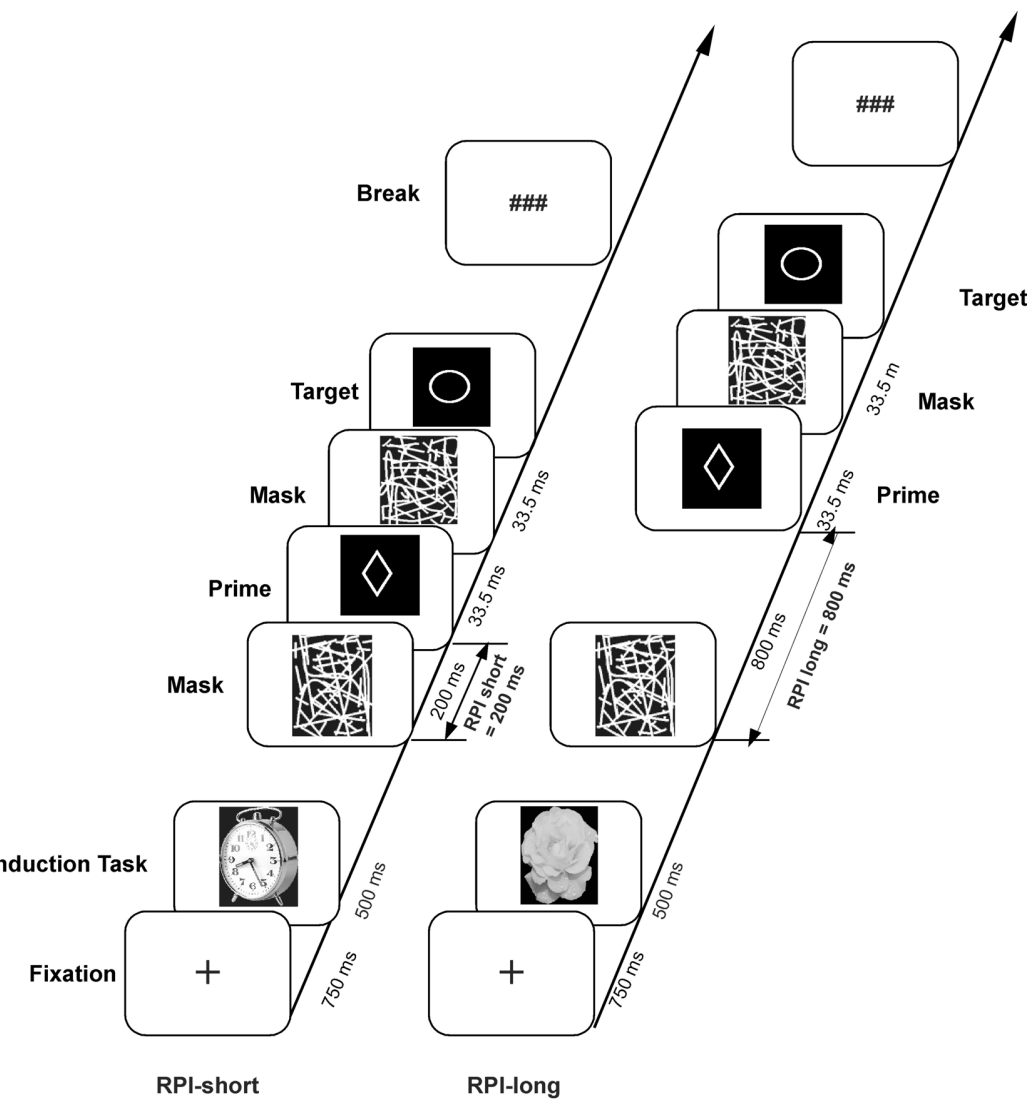

Semantic or Perceptual Induction Task

B

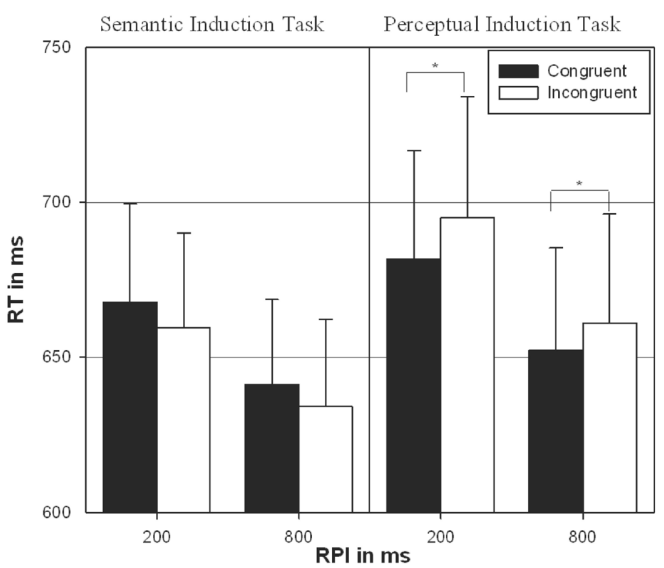

FIGURE 1 | (A) Induction task paradigm in combination with a masked visuo-motor priming task. The masked prime shape was presented either $200 \mathrm{~ms}$ or $800 \mathrm{~ms}$ following the response to the induction task (response prime interval, RPI) that is intended to elicit the corresponding task set. The $\mathrm{RPI}$ was varied in order to capture the temporal dynamics of task set activation. The semantic induction task required semantic classification (forced choice living/non-living decision) of the inducing picture, whereas the perceptual induction task required a forced choice perceptual classification decision of the pictured object (round/elongated shape). Subsequent to masked prime presentation, the target shape was presented, which required a right- or left-hand response. In the congruent priming condition, prime and target shape afforded the same manual response whereas in the incongruent condition, prime and target were associated with different response hands. (B) Behavioral unconscious visuo-motor priming effects of Exp. 2 of the Martens et al. (2011) study. Mean correct response times and their standard error in the target shape discrimination task, as a function of response congruency, induction task, and RPI. The asterisks indicate significant masked priming effects (difference between incongruent and congruent prime-target responses) within each induction task/RPI combination. Significant unconscious visuo-motor priming effects were only obtained following the perceptual induction task, but not following the semantic induction task. Modified after Kiefer and Martens (2010) and after Martens et al. (2011). 


\section{UNCONSCIOUS SEMANTIC PROCESSING}

Semantic processing of conceptual information provides the basis for thought, problem solving and action planning (for recent overviews see, Kiefer and Pulvermüller, 2011; Kiefer and Barsalou, 2012) and is also crucial for language and communication because it constitutes word meaning (Levelt et al., 1999). Semantic processing is, therefore, typically characterized as a higher-level cognitive process compared with more lowerlevel perceptually based visuo-motor processes (Engelkamp and Zimmer, 1994; Anderson, 2000). Nevertheless, there are meanwhile numerous demonstrations that semantic meaning is also processed unconsciously (e.g., Marcel, 1983; Carr and Dagenbach, 1990; Draine and Greenwald, 1998; Dell' Acqua and Grainger, 1999; Kiefer and Spitzer, 2000; Rolke et al., 2001; Kiefer, 2002; e.g., Kiefer and Brendel, 2006). Unconscious semantic processing can be demonstrated with the masked semantic priming paradigm. Semantic priming generally refers to a facilitation of a response to a meaningful target (e.g., word or picture), when it is preceded by a semantically related masked prime (Neely, 1977, 1991). For instance, lexical (word/pseudoword) decisions on word targets are faster, when they are preceded by a semantically related prime word (e.g., "chair-table") compared with unrelated pairings (e.g., "car-hen"). In contrast to visuo-motor priming, primes in the semantically related and unrelated conditions always afford the same response in the target task (word response in the lexical decision task) thereby ruling out any response congruency effects. In masked semantic priming, a pattern mask prevents conscious identification of the prime word. Nevertheless, responses to targets that have been preceded by a semantically related prime are performed more quickly than responses to targets paired with unrelated primes demonstrating unconscious access to word meaning (Carr and Dagenbach, 1990; Kiefer and Spitzer, 2000; Kiefer, 2002; Kiefer and Brendel, 2006). Neuroimaging (Mummery et al., 1999) and electrophysiological studies (Nobre and McCarthy, 1995; Kiefer et al., 2007) show that semantic priming depends on anterior temporal areas (ventral pathways) supporting semantic integration (Kiefer and Pulvermüller, 2011). Semantic priming modulates the N400 ERP component, a negative deflection peaking at about $400 \mathrm{~ms}$ with centro-parietal topography (Kutas and Hillyard, 1980). The N400 semantic priming effect is reflected by an attenuated N400 amplitude (i.e., relatively less negative voltage) to a target when preceded by a semantically related as compared with an unrelated prime (Bentin et al., 1985; Kiefer et al., 1998). Intracranial ERP recordings (Nobre and McCarthy, 1995) and source analyses of scalp potentials (Kiefer et al., 2007) have implicated a region in the anterior-ventral temporal lobe in generating the N400 ERP component.

There is meanwhile accumulating evidence that similar to visuo-motor priming unconscious semantic priming is modulated by executive control factors (for a recent review, see Kiefer et al., 2012). In fact most of the empirical tests of the attentional sensitization model of unconscious cognition with the induction task paradigm described above have been conducted within the field of unconscious semantic processing.

Similar to conscious semantic priming (for a review see, Deacon and Shelley-Tremblay, 2000), unconscious semantic priming has been shown to depend on attentional resources: in a masked semantic priming study within a lexical decision task (word/non-word decision) (Kiefer and Brendel, 2006), an attentional cue prompted participants to attend to the stimulation stream either during the time window of masked prime presentation or already one second earlier. In the latter long cue prime interval condition, subjects already had disengaged attention when the masked prime was finally presented. Kiefer and Brendel (2006) obtained a subliminal semantic priming effect on the N400 ERP component, but only when the masked prime was presented within the time window of attention. Hence, comparable to the findings in visuo-motor priming (Naccache et al., 2002), subliminal semantic priming depends on temporal attention. Furthermore, masked semantic priming was significantly reduced when the masked prime was preceded by a difficult primary task requiring greater attentional resources compared with an attentionally undemanding task (Martens and Kiefer, 2009). These influences of attentional capacity on unconscious semantic processing are in line with our proposal (Kiefer and Martens, 2010) that attention and conscious experience are functionally independent to some extent and should not be equated (see also, Koch and Tsuchiya, 2007; see also, Van Boxtel et al., 2010).

Using the induction task paradigm already described above, we systematically investigated the influence of activated task sets on subsequent masked semantic priming (Kiefer and Martens, 2010; Martens et al., 2011). It was assessed whether a semantic task set induced by a semantic decision task (e.g., living/non-living decision) immediately before masked prime presentation sensitizes semantic processing pathways and enhances subliminal semantic priming (see Figure 2A). In contrast, a perceptual task set induced by a task that requires attention to visual stimulus features (letter or object shape decision) should desensitize semantic pathways and therefore, attenuate subsequent subliminal semantic priming. The time interval between the response to the induction task and the onset of the prime (RPI) (either 200 or $800 \mathrm{~ms}$ ) was varied in order to obtain information on how the influence of the induction task on masked priming unfolds over time. We expected that a semantic induction task sensitizes semantic processing pathways and thus enhances semantic priming only at the short RPI (200 ms) because the task switching literature suggests that a task representation is active for about $600 \mathrm{~ms}$ after task completion (Rogers and Monsell, 1995), but is actively inhibited thereafter (Mayr and Keele, 2000).

Across experiments, the difficulty of the semantic and perceptual induction tasks as well as their verbal or non-verbal nature was systematically varied. For instance, in one experiment (Kiefer and Martens, 2010), participants performed an easy semantic word classification task (living/non-living decision) and a difficult perceptual letter classification task with words (first or last letter of a word has a closed or open shape). In other experiments (Kiefer and Martens, 2010; Martens et al., 2011), non-verbal induction tasks, which exhibited the same level of difficulty, required semantic classification (living/non-living decision) vs. perceptual classification (round vs. elongated shape decision) of object pictures (see Figure 2A).

Comparable results were obtained regardless of the difficulty level and the verbal or non-verbal nature of the induction tasks 
A

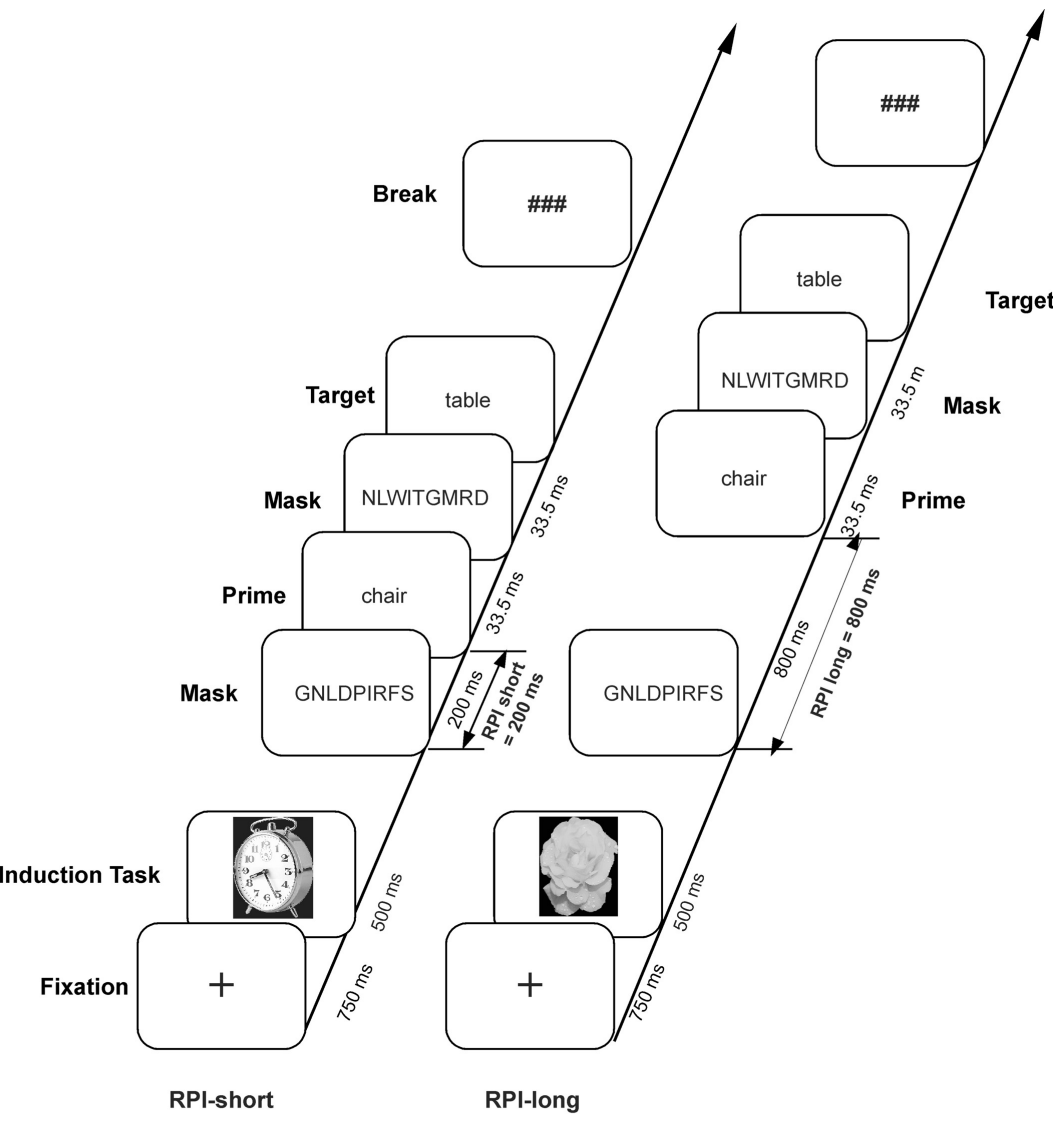

Semantic or Perceptual Induction Task

B

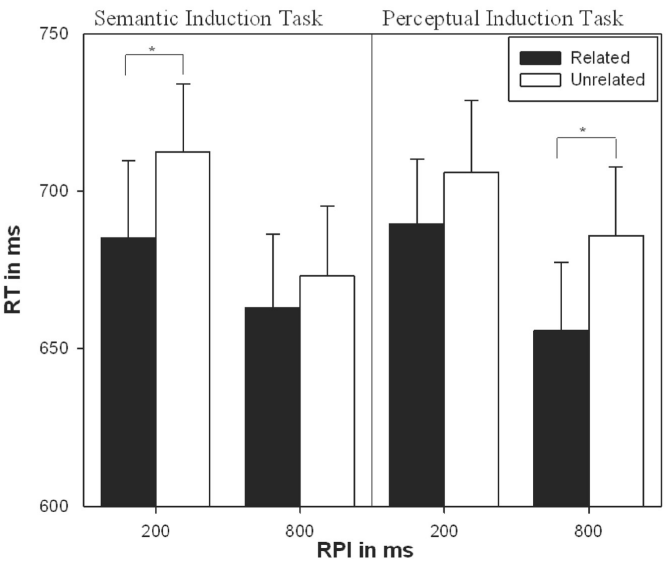

FIGURE 2 | (A) Induction task paradigm in combination with a masked semantic priming task. The masked prime word was presented either $200 \mathrm{~ms}$ or $800 \mathrm{~ms}$ following the response to the induction task (RPI). The semantic induction task required semantic classification (living/non-living decision) of the pictured object, whereas the perceptual induction task required a perceptual classification of the object (round/elongated shape). Subsequent to masked prime presentation, the target word was presented, which required a lexical decision (word/pseudoword). In the related priming condition, prime and target were semantically related ("table-chair") whereas in the unrelated condition, prime and target were semantically unrelated ("hen-car"). (B) Behavioral semantic priming effects of Exp. 1 of the Martens et al. (2011) study. Mean correct response times and their standard error in the lexical decision task, as a function of semantic relatedness, induction task, and RPI. The asterisks indicate significant masked priming effects (difference between semantically unrelated and related conditions) within each induction task/RPI combination. At the short RPI when the task sets were active, significant unconscious semantic priming effects were only obtained following the semantic induction task, but not following the perceptual induction task. At the long RPI when the task sets were inhibited, the opposite pattern was observed: unconscious semantic priming effects were only obtained following the perceptual induction task Modified after Kiefer and Martens (2010) and after Martens et al. (2011). 
(see Figure 2B for one example). At the short RPI, behavioral and electrophysiological semantic priming effects were obtained when a semantic task set was induced immediately before subliminal prime presentation, whereas a previously induced perceptual task set attenuated priming. In line with the attentional sensitization model, unconscious semantic processing is enhanced by a semantic and attenuated by a perceptual task set. At the long RPI, significant priming was found after the perceptual induction task, but not after the semantic task. This pattern of priming effects at the long RPI suggests that after $800 \mathrm{~ms}$ the task set of the induction task had been abandoned and a reconfiguration of the cognitive system in preparation for the upcoming lexical task had taken place (Kiefer and Martens, 2010): semantic pathways are sensitized when the perceptual induction task has been abandoned, but they are desensitized when the semantic induction task has been abandoned. This resulting pattern is compatible with the notion of a backward inhibition mechanism that suppresses irrelevant task sets in preparation of the next task (Mayr and Keele, 2000; Houghton et al., 2009). In line with this interpretation, the RPI did not modulate induction task influences on subliminal semantic priming when the task set of the induction task had to be maintained until the response to the lexical decision task was given (Kiefer, unpublished data). The differential modulatory effects of induction tasks on masked priming as a function of the RPI provide insight in the dynamic nature of cognitive reorganization during task set switching that in turn influences unconscious information processing.

A further recent study using the induction task paradigm examined the attentional boundary conditions for unconscious semantic priming to occur (Adams and Kiefer, submitted). It was assessed whether phonological task sets that are non-semantic in their nature, but do require some form of linguistic processing also reduce unconscious semantic priming. In particular, we were interested whether the effects of phonological induction tasks on subsequent semantic priming were comparable whether the focus was set on phonological processing of the entire word vs. single letters. Before the subliminally primed lexical decision task was presented, participants performed semantic and phonological induction tasks that should either activate a semantic or a phonological task set. Across two experiments, the nature of the phonological induction task (word phonology vs. letter phonology) was varied to assess whether the attentional focus on the entire word vs. single letters modulates subsequent masked semantic priming. In both experiments, subliminal semantic priming was only found subsequent to the semantic induction task, but was abolished following either phonological induction task. The results of this study indicate that attention to phonology attenuates subsequent semantic processing of unconsciously presented primes whether or not attention is directed to the entire word or to single letters. In line with the attentional sensitization model, these findings substantiate the notion that an attentional orientation toward semantics is necessary for unconscious semantic processing to be elicited.

This research on attentional influences on subliminal semantic priming helps to reconcile previous discrepant evidence regarding the automaticity of semantic processing. It has been argued that semantic processing is not automatic, but requires controlled access to conceptual meaning (Henik et al., 1994; Duscherer and Holender, 2002) because semantic priming with visible stimuli has been found to depend on the attentional orientation toward the prime word (for a review, see Maxfield, 1997; for a review, see Deacon and Shelley-Tremblay, 2000): earlier studies on the effects of prime tasks observed reduced or absent semantic priming when participants were required to attend to perceptual letter features of the prime (e.g., a letter search task) and not to its meaning (e.g., Chiappe et al., 1996; Mari-Beffa et al., 2005). These findings were taken as evidence that access to conceptual meaning is confined to a controlled processing mode. However, several other studies demonstrating that unconsciously perceived prime words can elicit semantic priming effects favor the view that semantic processing can also occur in an automatic fashion (Carr and Dagenbach, 1990; Draine and Greenwald, 1998; Kiefer and Spitzer, 2000; Rolke et al., 2001; Kiefer, 2002). This apparent contradiction can be easily resolved if one assumes that unconscious automatic processes depend on executive control through attentional sensitization (Kiefer and Martens, 2010). Our work using the induction task paradigm in combination with a subliminally primed lexical decision task (e.g., Kiefer and Martens, 2010; Martens et al., 2011) shows that even under purely automatic processing conditions, semantic priming is susceptible to executive control as predicted by our attentional sensitization model.

\section{CONCLUSIONS AND FUTURE RESEARCH DIRECTIONS}

Accumulating evidence demonstrates that various forms of unconscious processing are susceptible to executive control similar to conscious processing: subliminal visuo-motor, and semantic priming effects, prototypical examples of automatic processes, are modulated by executive control factors such as attentional resources, stimulus expectations, action intentions, and task sets. These top-down factors are typically considered to involve executive control mechanisms dedicated to organize action and thought (Norman and Shallice, 1986; Posner and DiGirolamo, 1998). Hence, in contrast to classical theories of automaticity and executive control (Posner and Snyder, 1975; Schneider and Shiffrin, 1977), automatic processes elicited by unconscious visual stimuli are under executive control to some extent. The findings reviewed here support refined theories of automaticity (Neumann, 1990; Naccache et al., 2002; Moors and De Houwer, 2006) in general and the attentional sensitization model of unconscious information processing in particular (Kiefer, 2007; Kiefer and Martens, 2010). According to the attentional sensitization model (Kiefer and Martens, 2010), processing can occur automatically in the sense that it does not depend on conscious awareness and that it is initiated without deliberate intention. However, unconscious automatic processing is modulated by executive control and depends on an appropriate configuration of the cognitive system. Thus, within the light of the attentional sensitization model and the data reviewed in this article, unconscious automatic processing and the notion of executive control are compatible. As shown above, the assumption of attentional sensitization of unconscious information processing can accommodate conflicting findings regarding the automaticity of processes in many areas of psychology and the neurosciences such as visuo-motor (Bub and Masson, 2010), and semantic processing 
(Rees et al., 1999; Kiefer and Martens, 2010). The attentional sensitization mechanism may thus apply to many domains and has the explanatory power to account for seemingly contradictory empirical phenomena. Evidence for executive control over unconscious visuo-motor and semantic processing described in the previous sections concerned neuro-cognitive functions predominantly processed in cortical structures. However, if attentional sensitization of unconscious information processing is a general principle, it should also apply to emotional processes, which depend at least in part on subcortical structures (LeDoux, 1996; Rolls, 1999).

\section{IS UNCONSCIOUS EMOTIONAL PROCESSING MODULATED BY EXECUTIVE CONTROL?}

Unconscious emotional processes can be investigated with the masked affective priming paradigm. In affective priming, which is assumed to depend on activation of emotional representations, primes and targets (words or pictures) share the same or different (positive, e.g., baby vs. negative, e.g., shark) emotional valence (Fazio et al., 1986; Fazio, 2001). For instance, in an evaluative judgment task (pleasant vs. unpleasant) on visible target stimuli, responses are typically faster when primes and targets exhibit the same emotional valence (for review, see Fazio, 2001). Affective priming activated emotional brain areas including the amygdala, insula, and orbitofrontal cortex (Liu et al., 2010). Although visuo-motor response conflict certainly plays a role for emotional priming during evaluative judgments (Klauer et al., 1997; Klinger et al., 2000), the use of tasks with response-categories that differ from prime-target relatedness demonstrated the involvement of emotional processes in addition to response-based processes (Spruyt et al., 2007; Wentura and Frings, 2008). It should be noted that in most of these experiments stimuli were presented visibly. Nevertheless, these findings are suggestive for executive control influences on unconscious emotional processing as it is shown below.

In the literature on affective priming, there has been a longstanding debate whether emotional information is automatically processed. This debate has been fueled by the observation that affective priming with visible (e.g., Klauer and Musch, 2001; Spruyt et al., 2004) or subliminal primes (Eckstein and Perrig, 2007) frequently disappeared when target tasks other than evaluative judgments (e.g., semantic categorization or word pronunciation) were used. For instance, affective congruency (positive vs. negative valence) of subliminally presented masked prime words elicited only priming effects on the subsequent target decision during an evaluative judgment task, but not during a non-emotional semantic (living/non-living) judgment (Eckstein and Perrig, 2007). Unconscious automatic emotional processing has, therefore, been considered to be a spurious phenomenon (for a discussion, Klinger et al., 2000; for a discussion, Klauer and Musch, 2003). This conclusion is premature, however, because within the light of the attentional sensitization model (Kiefer and Martens, 2010) even unconscious automatic emotional processing depends on an attentional amplification of corresponding processing pathways. When the target task requires attention to non-emotional semantic stimulus features (e.g., living/nonliving decision), emotional pathways are desensitized resulting in decreased or absent emotional priming. Hence, for emotional priming to occur, attention must be directed to the emotional stimulus dimension in order to sensitize emotional processing pathways (Spruyt et al., 2009, 2012).

Spruyt and colleagues (Spruyt et al., 2009, 2012) tested the proposed attentional modulation of emotional processing in two studies. In all experiments, emotional priming was probed during a word pronunciation task, which did not explicitly require emotional processing. With such a pronunciation task most previous studies failed to find emotional priming effects (for a review see, Klauer and Musch, 2003). Most critically, in the experiments by Spruyt and colleagues attention was directed to emotional or non-emotional semantic word features in separate participant groups by interspersing different types of induction trials between the priming trials requiring word pronunciation: these induction trials required an emotional or a non-emotional semantic categorization of words and should differentially allocate attention to emotional vs. non-emotional semantic features. Based on the assumption that automatic emotional processing requires an attentional sensitization of emotional pathways, emotional priming during a pronunciation task should only be obtained within the context of emotional induction trials, but not within the context of non-emotional semantic induction trials. Results clearly confirmed these predictions: whether primes were presented unmasked and visibly (Spruyt et al., 2009) or masked and unconsciously (Spruyt et al., 2012), affective congruency between primes and targets facilitated target pronunciation only in the blocks with the emotional induction trials. These findings provide supporting evidence for an attentional modulation of unconscious emotional processing and help to reconcile discrepant findings regarding the automaticity of emotional priming: emotional priming whether elicited by conscious or unconscious stimuli, crucially requires a sensitization of emotional pathways. Thus, attentional sensitization of unconscious processing may apply to emotional brain circuits, which partially involve subcortical structures (LeDoux, 1996; Rolls, 1999), and is not restricted to predominant cortical processes such as visuo-motor or semantic processing. However, as data regarding attentional modulation of unconscious emotional processing are scarce, this research area certainly deserves further investigation.

\section{FLEXIBILITY OF UNCONSCIOUS PROCESSING IN HEALTHY AND CLINICAL POPULATIONS}

Our general experimental approach that combines a first task for inducing task sets with a subsequent (subliminally) primed decision task is a useful tool for addressing many research questions regarding executive control of unconscious information processing. It can be used to test whether other forms of unconscious processes than presently investigated in detail (e.g., emotional, visuo-spatial, motor, phonological, orthographic) are similarly susceptible to executive control. It would also be interesting to investigate whether attentional sensitization applies to implicit memory (Schacter, 1995) or implicit learning (Curran, 1998). This would demonstrate that attentional sensitization of unconscious information processing is a general mechanism implemented in the human neuro-cognitive system. The notion of attentional sensitization of unconscious information processing 
could also help to explain and to further empirically investigate cognitive impairments in clinical populations who occasionally show deficits in unconscious information processing (Kiefer et al., 2009). In particular, conscious control of unconscious emotional processing is clinically highly important because findings in this area might help to design more efficient therapeutic treatment techniques for mood and anxiety disorders. The present approach could also help to elucidate why putatively automatic cognitive processes are strongly modulated by hypnotic induction. For instance, the Stroop interference effect (Stroop, 1935) that depends on a conflict between task-irrelevant automatic processes of word reading and task-relevant processes of color naming (Cohen et al., 1990) is abolished when participants receive the hypnotic suggestion that (English) color words should be conceived as meaningless character strings written in an unknown alphabet (Raz et al., 2006, 2007). Hence, our framework could contribute to a better understanding of the attentional mechanisms underlying the effects of hypnosis in research and therapeutic settings.

Although much progress has been made to demonstrate executive control influences on unconscious information processing using behavioral or neurophysiological measures, its precise functional-anatomical architecture has to be better characterized: the attentional sensitization model assumes that control of

\section{REFERENCES}

Anderson, J. R. (2000). Learning and Memory: An Integrated Approach. New York, NY: John Wiley and Sons.

Ansorge, U., Fuchs, I., Khalid, S., and Kunde, W. (2011a). No conflict control in the absence of awareness. Psychol. Res. 75, 351-365.

Ansorge, U., Heumann, M., and Scharlau, I. (2002). Influences of visibility, intentions, and probability in a peripheral cuing task. Conscious. Cogn. 11, 528-545.

Ansorge, U., and Horstmann, G. (2007). Preemptive control of attentional capture by colour: evidence from trial-by-trial analyses and orderings of onsets of capture effects in reaction time distributions. Q. J. Exp. Psychol. 60, 952-975.

Ansorge, U., Horstmann, G., and Scharlau, I. (2011b). Top-down contingent feature-specific orienting with and without awareness of the visual input. Adv. Cogn. Psychol. 7, 108-119.

Ansorge, U., Kiefer, M., Khalid, S., Grassl, S., and König, P. (2010). Testing the theory of embodied cognition with subliminal words. Cognition 116, 303-320.

Ansorge, U., Kiss, M., and Eimer, M. (2009). Goal-driven attentional capture by invisible colours. Psychon. Bull. Rev. 16, 648-653.

Ansorge, U., and Neumann, O. (2005). Intentions determine the effect of invisible metacontrast-masked contingencies in a peripheral cueing task. J. Exp. Psychol. Hum. Percept. Perform. 31, 762-777.

Bargh, J. A. (1989). "Conditional automaticity: varieties of automatic influence in social perception and cognition," in Unintended Thought, eds J. S. Uleman and J. A. Bargh (New York, NY: Guilford Press), 3-51.

Bentin, S., McCarthy, G., and Wood, C. C. (1985). Event-related potentials, lexical decision, and semantic priming. Electroencephalogr. Clin.

Bode, S., and Haynes, J. D. (2008). Decoding sequential stages of task preparation in the human brain. Neuroimage 45, 606-613.

Botvinick, M. M., Braver, T. S., Barch, D. M., Carter, C. S., and Cohen, J. D. (2001). Conflict monitoring and cognitive control. Psychol. Rev. 108, 624-652.

Boucart, M., Humphreys, G. W., and Lorenceau, J. (1995). Automatic access to object identity: attention to global information, not to particular physical dimensions, is important. J. Exp. Psychol. Hum. Percept. Perform. 21, 584-601.

Breitmeyer, B. G., and Öğmen, H. (2006). Visual Masking: Time Slices Through Conscious and Unconscious Vision. Oxford: Oxford University Press. primes: evidence for top-down Neurophysiol. 60, 343-355.

unconscious processes is exerted by a prefrontal top-down signal, which influences the sensitivity in brain circuits that process specific information such as stimulus dimensions or features. This assumption can be tested in more detail by means of fMRI and electrophysiological recording techniques.

In conclusion, the executive control influences of unconscious processing reviewed here demonstrates the adaptability of the cognitive system in optimizing ongoing processing toward the pursuit of an intended goal. This research suggests that preemptive executive control of unconscious processes as postulated by the attentional sensitization model coordinates even the unconscious processing streams in congruency with higherlevel task representations. This considerably reduces the effort of the cognitive control system to organize behavior because taskincongruent processes are dampened at relatively early stages. Hence, attentional sensitization of unconscious information processing contributes to an effective goal-related adaptation of our cognitive system.

\section{ACKNOWLEDGMENTS}

This research was supported by a grant of the German Research Foundation (DFG Ki 804/3-2) within the Research Network "Neuro-Cognitive Mechanisms of Conscious and Unconscious Visual Perception" (PAK 270/2) to Markus Kiefer.

Bub, D. N., and Masson, M. E. J. (2010). Grasping beer mugs: on the dynamics of alignment effects induced by handled objects. J. Exp. Psychol. Hum. Percept. Perform. 36, 341-358.

Carr, T. H., and Dagenbach, D. (1990). Semantic priming and repetition priming from masked words: evidence for a center-surround attentional mechanism in perceptual recognition. J. Exp. Psychol. Learn. Mem. Cogn. 16, 341-350.

Chiappe, P. R., Smith, M. C., and Besner, D. (1996). Semantic priming in visual word recognition: activation blocking and domains of processing. Psychon. Bull. Rev. 3, 249-253.

Cohen, J. D., Dunbar, K., and McClelland, J. L. (1990). On the control of automatic processes: a parallel distributed processing account of the Stroop effect. Psychol. Rev. 97, 332-361.

Curran, T. (1998). "Implicit sequence learning from a cognitive neuroscience perspective," in Handbook of Implicit Learning, eds M. A. Stadler and P. A. Frensch (Thousand Oaks, CA: Sage), 365-400.

Deacon, D., and Shelley-Tremblay, J. (2000). How automatically is meaning accessed: a review of the effects of attention on semantic processing. Front. Biosci. 5, E82-E94.

Dehaene, S., Changeux, J. P., Naccache, L., Sackur, J., and Sergent, C. (2006). Conscious, preconscious, and subliminal processing: a testable taxonomy. Trends Cogn. Sci. 10, 204-211.

Dehaene, S., and Naccache, L. (2001). Towards a cognitive neuroscience of consciousness: basic evidence and a workspace framework. Cognition 79, 1-37.

Dehaene, S., Naccache, L., Leclec'h, G., Koechlin, E., Mueller, M., DehaeneLambertz, G., Van De Moortele, P.-F., and Lebihan, D. (1998). Imaging unconscious priming. Nature 395, 597-600.

Dell' Acqua, R., and Grainger, J. (1999). Unconscious semantic priming from pictures. Cognition 73, B1-B15.

Draine, S. C., and Greenwald, A. G. (1998). Replicable unconscious semantic priming. J. Exp. Psychol. Gen. 127, 286-303.

Duscherer, K., and Holender, D. (2002). No negative semantic priming from unconscious flanker words in sight. J. Exp. Psychol. Hum. Percept. Perform. 28, 839-853.

Eckstein, D., and Perrig, W. J. (2007). The influence of intention on masked priming: a study with semantic classification of words. Cognition 104, 345-376.

Engelkamp, J., and Zimmer, H. D. (1994). The Human Memory. A Multi-Modal Approach. Seattle, WA: Hogrefe and Huber.

Fazio, R. H. (2001). On the automatic activation of associated evaluations: 
an overview. Cogn. Emot. 15, 115-141.

Fazio, R. H., Sanbonmatsu, D. M., Powell, M. C., and Kardes, F. R. (1986). On the automatic activation of attitudes. J. Pers. Soc. Psychol. 50, 229-238.

Greenwald, A. G., Draine, S. C., and Abrams, R. L. (1996). Three cognitive markers of unconscious semantic activation. Science 273, 1699-1702.

Hamker, F. H. (2005). The reentry hypothesis: the putative interaction of the frontal eye field, ventrolateral prefrontal cortex, and areas V4, IT for attention and eye movement. Cereb. Cortex 15, 431-447.

Haynes, J. D., Sakai, K., Rees, G., Gilbert, S., Frith, C., and Passingham, R. E. (2007). Reading hidden intentions in the human brain. Curr. Biol. 17, 323-328.

Henik, A., Friedrich, F. J., Tzelgov, J., and Tramer, S. (1994). Capacity demands of automatic processes in semantic priming. Mem. Cognit. 22, 157-168.

Hopfinger, J. B., Buonocore, M. H., and Mangun, G. R. (2000). The neural mechanisms of top-down attentional control. Nat. Neurosci. 3, 284-291.

Hopfinger, J. B., Woldorff, M. G., Fletcher, E. M., and Mangun, G. R. (2001). Dissociating topdown attentional control from selective perception and action. Neuropsychologia 39, 1277-1291.

Houghton, G., Pritchard, R., and Grange, J. A. (2009). The role of cue-target translation in backward inhibition of attentional set. J. Exp. Psychol. Learn. Mem. Cogn. 35, 466-476.

Jaśkowski, P., Skalska, B., and Verleger, R. (2003). How the self-controls its "automatic pilot" when processing subliminal information. J. Cogn. Neurosci. 15, 911-920.

Kiefer, M. (2002). The N400 is modulated by unconsciously perceived masked words: further evidence for an automatic spreading activation account of N400 priming effects. Cogn. Brain Res. 13, 27-39.

Kiefer, M. (2007). Top-down modulation of unconscious 'automatic' processes: a gating framework. $A d v$. Cogn. Psychol. 3, 289-306.

Kiefer, M., Adams, S. C., and Zovko, M. (2012). Attentional sensitization of unconscious visual processing: top-down influences on masked priming. Adv. Cogn. Psychol. 8, 50-61.

Kiefer, M., Ansorge, U., Haynes, J. D., Hamker, F., Mattler, U., Verleger, R., and Niedeggen, M. (2011).
Neuro-cognitive mechanisms of conscious and unconscious visual perception: from a plethora of phenomena to general principles. Adv. Cogn. Psychol. 7, 55-67.

Kiefer, M., and Barsalou, L. W. (2012). "Grounding the human conceptual system in perception, action, and internal states," in Tutorials in Action Science, eds W. Prinz, M. Beisert, and A. Herwig (Cambridge: MIT Press), (in press).

Kiefer, M., and Brendel, D. (2006). Attentional modulation of unconscious 'automatic' processes: evidence from event-related potentials in a masked priming paradigm. $J$. Cogn. Neurosci. 18, 184-198.

Kiefer, M., and Martens, U. (2010). Attentional sensitization of unconscious cognition: task sets modulate subsequent masked semantic priming. J. Exp. Psychol. Gen. 139, 464-489.

Kiefer, M., Martens, U., Weisbrod, M., Hermle, L., and Spitzer, M. (2009). Increased unconscious semantic activation in schizophrenia patients with formal thought-disorder. Schizophr. Res. 114, 79-83.

Kiefer, M., and Pulvermüller, F. (2011). Conceptual representations in mind and brain: theoretical developments, current evidence and future directions. Cortex. doi: 10.1016/j.cortex.2011.04.006. [Epub ahead of print].

Kiefer, M., Schuch, S., Schenck, W., and Fiedler, K. (2007). Mood states modulate activity in semantic brain areas during emotional word encoding. Cereb. Cortex 17, 1516-1530.

Kiefer, M., and Spitzer, M. (2000). Time course of conscious and unconscious semantic brain activation. Neuroreport 11, 2401-2407.

Kiefer, M., Weisbrod, M., Kern, I., Maier, S., and Spitzer, M. (1998). Right hemisphere activation during indirect semantic priming: evidence from event-related potentials. Brain Lang. 64, 377-408.

Kiesel, A., Kunde, W., Pohl, C., Berner, M. P., and Hoffmann, J. (2009). Playing chess unconsciously. J. Exp. Psychol. Learn. Mem. Cogn. 35, 292-298.

Kiesel, A., Kunde, W., Pohl, C., and Hoffmann, J. (2006). Priming from novel masked stimuli depends on target set size. Adv. Cogn. Psychol. 2, $37-45$.

Kiesel, A., Steinhauser, M., Wendt, M., Falkenstein, M., Jost, K., Philipp, A. M., and Koch, I. (2010). Control and inference in task switching - A review. Psychol. Bull. 136, 849-874.

Klauer, K. C., and Musch, J. (2001). Does sunshine prime loyal?
Affective priming in the naming task. Q. J. Exp. Psychol. A 54, 727-751.

Klauer, K. C., and Musch, J. (2003). "Affective priming: findings and theories," in The Psychology of Evaluation: Affective Processes in Cognition and Emotion, eds J. Musch and K. C. Klauer (Mahwah, NJ: Lawrence Erlbaum Associates, Inc.), 7-49.

Klauer, K. C., Rossnagel, C., and Musch, J. (1997). List-context effects in evaluative priming. $J$. Exp. Psychol. Learn. Mem. Cogn. 23, 246-255.

Klinger, M. R., Burton, P. C., and Pitts G. S. (2000). Mechanisms of unconscious priming: I. Response competition, not spreading activation. $J$. Exp. Psychol. Learn. Mem. Cogn. 26, 441-455.

Koch, C., and Tsuchiya, N. (2007) Attention and consciousness: two distinct brain processes. Trends Cogn. Sci. 11, 16-22.

Kunde, W., Kiesel, A., and Hoffmann, J. (2003). Conscious control over the content of unconscious cognition. Cognition 88, 223-242.

Kunde, W., Reuss, H., and Kiesel, A. (2012). Consciousness and cognitive control. Adv. Cogn. Psychol. 8, 9-18.

Kutas, M., and Hillyard, S. A. (1980). Reading senseless sentences: brain potentials reflect semantic incongruity. Science 207, 203-205.

LeDoux, G. E. (1996). The Emotional Brain. New York, NY: Simon and Schuster.

Levelt, W. J., Roelofs, A., and Meyer, A. S. (1999). A theory of lexical access in speech production. Behav. Brain Sci. 22, 1-38; discussion 38-75.

Liu, H., Hu, Z., Peng, D., Yang, Y., and Li, K. (2010). Common and segregated neural substrates for automatic conceptual and affective priming as revealed by event-related functional magnetic resonance imaging. Brain Lang. 112 , $121-128$.

Logan, G. D. (1989). "Automaticity and cognitive control," in Unintended Thought, eds J. S. Uleman and J. A. Bargh (New York, NY: Guilford Press), 52-74.

Marcel, A. J. (1983). Conscious and unconscious perception: experiments on visual masking and word recognition. Cogn. Psychol. 15, 197-237.

Mari-Beffa, P., Valdes, B., Cullen, D. J., Catena, A., and Houghton, G. (2005). ERP analyses of task effects on semantic processing from words. Cogn. Brain Res. 23, 293-305.
Martens, U., Ansorge, U., and Kiefer, M. (2011). Controlling the unconscious: attentional task sets modulate subliminal semantic and visuo-motor processes differentially. Psychol. Sci. 22, 282-291.

Martens, U., and Kiefer, M. (2009). Specifying attentional top-down influences on subsequent unconscious semantic processing. $A d v$. Cogn. Psychol. 5, 56-68.

Mattler, U. (2003). Priming of mental operations by masked stimuli. Percept. Psychophys. 65, 167-187.

Mattler, U. (2005). Inhibition and decay of motor and non-motor priming. Percept. Psychophys. 67, 285-300.

Mattler, U. (2006). On the locus of priming and inverse priming effects. Percept. Psychophys. 68, 975-991.

Maxfield, L. (1997). Attention and semantic priming: a review of prime task effects. Conscious. Cogn. 6 204-218.

Mayr, U., and Keele, S. W. (2000). Changing internal constraints on action: the role of backward inhibition. J. Exp. Psychol. Gen. 129, 4-26.

Merikle, P. M., Joordens, S., and Stolz, J. A. (1995). Measuring the relative magnitude of unconscious influences. Conscious. Cogn. 4, 422-439.

Moors, A., and De Houwer, J. (2006). Automaticity: a theoretical and conceptual analysis. Psychol. Bul. 132, 297-326.

Mummery, C. J., Shallice, T., and Price, C. J. (1999). Dual-process model in semantic priming: a functional imaging perspective. Neuroimage 9 , 516-525.

Naccache, L., Blandin, E., and Dehaene, S. (2002). Unconscious masked priming depends on temporal attention. Psychol. Sci. 13, 416-424.

Neely, J. H. (1977). Semantic priming and retrieval from lexical memory: roles of inhibitionless spreading activation and limited-capacity attention. J. Exp. Psychol. 106 226-254.

Neely, J. H. (1991). "Semantic priming effects in visual word recognition: a selective review of current findings and theories," in Basic Progresses in Reading - Visual Word Recognition, eds D. Besner and G. W. Humphreys (Hillsdale, NJ: Lawrence Erlbaum Associates), 264-333.

Neumann, O. (1990). Direct parameter specification and the concept of perception. Psychol. Res. 52, 207-215.

Neumann, O., and Klotz, W. (1994). "Motor responses to nonreportable, masked stimuli: where is the limit of direct parameter specification?," in Attention and Performance 15: Conscious and Nonconscious 
Information Processing, eds C. Umiltá and M. Moscovitch (Cambridge, MA: The MIT Press), 123-150.

Nobre, A. C., and McCarthy, G. (1995). Language-related field potentials in the anterior-medial temporal lobe: II. Effects of word type and semantic priming. J. Neurosci. 15, 1090-1098.

Norman, D. A., and Shallice, T. (1986). "Attention to action: willed and automatic control of behavior," in Consciousness and Self-regulation, eds R. J. Davidson, G. E. Schwartz, and D. Shapiro (New York, NY: Plenum Press), 1-18.

Pessoa, L., Kastner, S., and Ungerleider, L. G. (2003). Neuroimaging studies of attention: from modulation of sensory processing to topdown control. J. Neurosci. 23, 3990-3998.

Pessoa, L., McKenna, M., Gutierrez, E., and Ungerleider, L. G. (2002). Neural processing of emotional faces requires attention. Proc. Natl. Acad. Sci. U.S.A. 99, 11458-11463.

Posner, M. I., and DiGirolamo, G. J. (1998). "Executive attention: conflict, target detection and cognitive control," in The Attentive Brain, ed R. Parasuman (Cambridge, MA: MIT Press), 401-423.

Posner, M. I., and Snyder, C. R. R. (1975). "Attention and cognitive control," in Information Processing and Cognition: The Loyola Symposium, ed R. L. Solso (Hillsdale, NJ: Lawrence Erlbaum Associates), 55-85.

Raz, A., Kirsch, I., Pollard, J., and Nitkin-Kaner, Y. (2006). Suggestion reduces the Stroop effect. Psychol. Sci. 17, 91-95.

Raz, A., Moreno-Iniguez, M., Martin, L., and Zhu, H. (2007). Suggestion overrides the Stroop effect in highly hypnotizable individuals. Conscious. Cogn. 16, 331-338.

Rees, G., Russell, C., Frith, C. D., and Driver, J. (1999). Inattentional blindness versus inattentional amnesia for fixated but ignored words. Science 286, 2504-2507.

Reuss, H., Kiesel, A., Kunde, W., and Hommel, B. (2011). Unconscious activation of task sets. Conscious. Cogn. 20, 556-567.

Reynolds, J. H., Pasternak, T., and Desimone, R. (2000). Attention increases sensitivity of V4 neurons. Neuron 26, 703-714

Rogers, R. D., and Monsell, S. (1995). Costs of a predictible switch between simple cognitive tasks. J. Exp. Psychol. Gen. 124, 207-231.

Rolke, B., Heil, M., Streb, J., and Henninghausen, E. (2001). Missed prime words within the attentional blink evoke an $\mathrm{N} 400$ semantic priming effect. Psychophysiology 38, 165-174.

Rolls, E. T. (1999). The Brain and Emotion. Oxford: Oxford University Press.

Schacter, D. L. (1995). "Implicit memory: a new frontier for cognitive neuroscience," in The Cognitive Neurosciences, ed M. S. Gazzaniga (Cambridge, MA: The MIT Press), 815-824.

Scharlau, I., and Ansorge, U. (2003). Direct parameter specification of an attention shift: evidence from perceptual latency priming. Vision Res. $43,1351-1363$.

Schmidt, F., Haberkamp, A., and Schmidt, T. (2011). Dos and don'ts in response priming research. $A d v$. Cogn. Psychol. 7, 120-131.

Schneider, W., and Shiffrin, R. M. (1977). Controlled and automatic human information processing: 1. Detection, search, and attention. Psychol. Rev. 84, 1-66.
Spruyt, A., De Houwer, J., Everaert, T., and Hermans, D. (2012). Unconscious semantic activation depends on feature-specific attention allocation. Cognition 122, 91-95.

Spruyt, A., De Houwer, J., and Hermans, D. (2009). Modulation of automatic semantic priming by feature-specific attention allocation. J. Mem. Lang. 61, 37-54.

Spruyt, A., De Houwer, J., Hermans, D. and Eelen, P. (2007). Affective priming of nonaffective semantic categorization responses. Exp. Psychol. 54 44-53.

Spruyt, A., Hermans, D., Pandelaere, M., De Houwer, J., and Eelen, P. (2004). On the replicability of the affective priming effect in the pronunciation task. Exp. Psychol. 51, 109-115.

Stroop, J. R. (1935). Studies of interference in serial verbal reactions. J. Exp. Psychol. 18, 643-662.

Tapia, E., Breitmeyer, B. G., and Shooner, C. R. (2010). Role of task-directed attention in nonconscious and conscious response priming by form and color. J. Exp. Psychol. Hum. Percept. Perform. 36, 74-87.

Treue, S., and Martínez Trujillo, J. C. (1999). Feature-based attention influences motion processing gain in macaque visual cortex. Nature 399, 575-579.

Van Boxtel, J. J. A., Tsuchiya, N., and Koch, C. (2010). Consciousness and attention: on sufficiency and necessity. Front. Psychol. 1:217. doi: 10.3389/fpsyg.2010.00217

Vorberg, D., Mattler, U., Heinecke, A., Schmidt, T., and Schwarzbach, J. (2003). Different time courses for visual perception and action priming. Proc. Natl. Acad. Sci. U.S.A. 100, 6275-6280.
Wentura, D., and Frings, C. (2008) Response-bound primes diminish affective priming in the naming task. Cogn. Emot. 22, 374-384.

Wiese, H., Schweinberger, S. R., and Neumann, M. F. (2008). Perceiving age and gender in unfamiliar faces: brain potential evidence for implicit and explicit person categorization. Psychophysiology 45, 957-969.

Wokke, M. E., Van Gaal, S., Scholte, H. S., Ridderinkhof, K. R., and Lamme, V. A. (2011). The flexible nature of unconscious cognition. PLoS One 6:e25729. doi: 10.1371/journal.pone.0025729

Wolbers, T., Schoell, E. D., Verleger, R., Kraft, S., McNamara, A., Jaśkowski, P., and Büchel, C. (2006). Changes in connectivity profiles as a mechanism for strategic control over interfering subliminal information. Cereb. Cortex 16, 857-864.

Conflict of Interest Statement: The author declares that the research was conducted in the absence of any commercial or financial relationships that could be construed as a potential conflict of interest.

Received: 06 February 2012; accepted: 09 March 2012; published online: 23 March 2012

Citation: Kiefer M (2012) Executive control over unconscious cognition: attentional sensitization of unconscious information processing. Front. Hum. Neurosci. 6:61. doi: 10.3389/fnhum. 2012.00061

Copyright (c) 2012 Kiefer. This is an open-access article distributed under the terms of the Creative Commons Attribution Non Commercial License, which permits non-commercial use, distribution, and reproduction in other forums, provided the original authors and source are credited. 